\title{
Recurrent Giant Prostatic Urethral Calculus: A Case Report and Mini-Review of the Literature
}

\author{
Sahbi Naouar ${ }^{\mathrm{a}, \mathrm{b}}$, Badreddine Ben Khalifa ${ }^{\mathrm{a}}$, Salem Braiek ${ }^{\mathrm{a}}$, Rafik El Kamel ${ }^{\mathrm{a}}$
}

\begin{abstract}
Prostatic urethral giant calculus is extremely unusual. A 37-year-old man was diagnosed with a giant prostatic urethral calculus; he underwent suprapubic cystotomy and the calculus was removed through a bladder neck incision. Recurrence of prostatic calculus was noted 2 years later. Urethroscopy screening was performed and revealed a giant calculus located partially in a prostatic diverticulum. Complete endoscopic removal was not feasible and calculus was extracted entirely via cystotomy through bladder neck. The calculus was calcium phosphates in composition.
\end{abstract}

Keywords: Prostate; Urethra; Calculus; Giant; Diverticulum; Recurrence

\section{Introduction}

Calculi in the urethra are an uncommon entity. Giant calculi in prostatic urethra are exceedingly rare, and less than 20 cases have been reported in the literature [1]. We report our experience with a case of recurrent giant prostatic calculus with prostatic diverticulum in a young male patient. The etiopathogenesis, diagnosis and suggested management of this rare pathology are detailed.

\section{Case Report}

A 37-year-old man, unmarried, presented with complaints of lower urinary tract symptoms (LUTS). He reported a 6-month history of dysuria, pollakiuria, and hypogastric pain with the feeling of incomplete emptying. On digital examination, stony hardness was noted through the anterior rectal wall. The rest of

\footnotetext{
Manuscript accepted for publication December 21, 2016

aUrology Department, Les Aghlabides Surgical Division, Ibn El Jazzar Teaching Hospital, Kairouan, Tunisia

'Corresponding Author: Sahbi Naouar, Urology Department, Les Aghlabides Surgical Division, Ibn El Jazzar Teaching Hospital, Kairouan 3100, Tunisia. Email: snaouar@laposte.net
}

the examination was unremarkable.

A kidney, ureter, and bladder (KUB) X-ray and abdominal ultrasound were performed and objectived bladder calculus of $6 \mathrm{~cm}$ without upper urinary tract calculi (Fig. 1), significant post void urine volume and thinking of the bladder wall. Laboratory studies showed urine culture grew Klebsiella pneumoniae. After antibiotic therapy, the patient underwent suprapubic cystotomy; calculi were located in the prostatic urethra and removed through bladder neck incision. At 3-month follow-up, the patient was free of symptoms. However, retrograde ejaculation was reported. Retrograde cystography objectived stone free large prostatic fossa and no urethral stenosis.

The patient was not compliant and presented again with LUTS, 2 years later. Retrograde cystography was performed and revealed recurrent $5 \mathrm{~cm}$ prostatic urethral calculus with a significant post void urine volume, large bladder neck and no

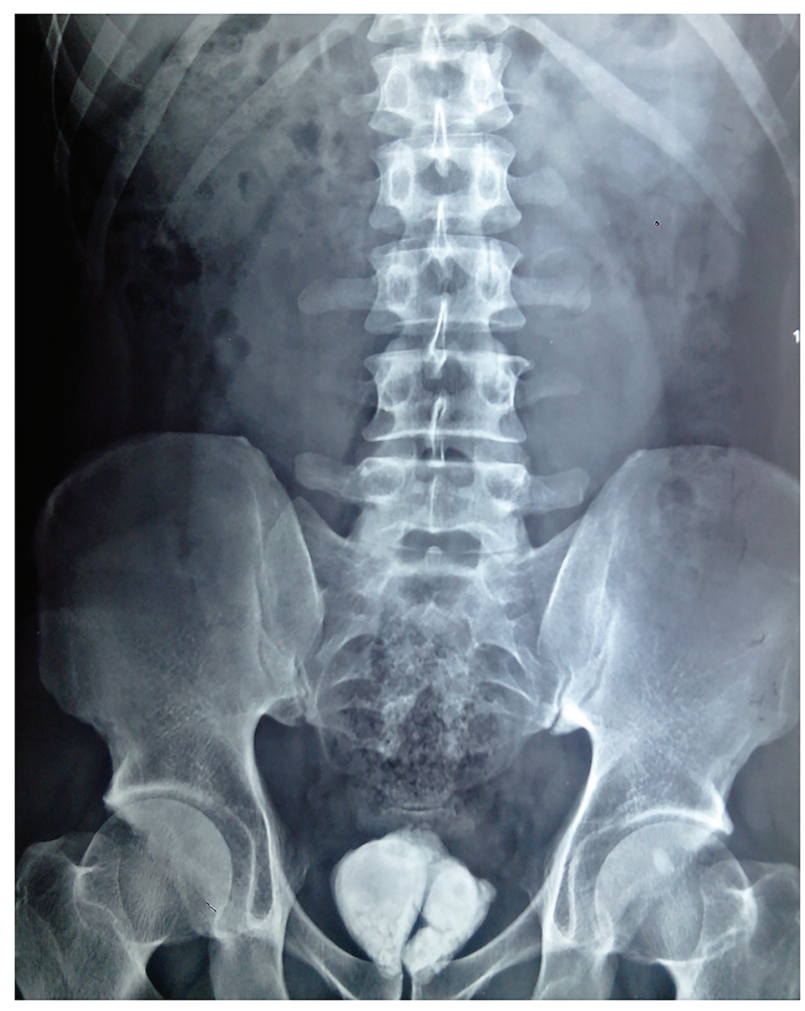

Figure 1. KUB X-ray showing the first prostatic urethral calculus. 


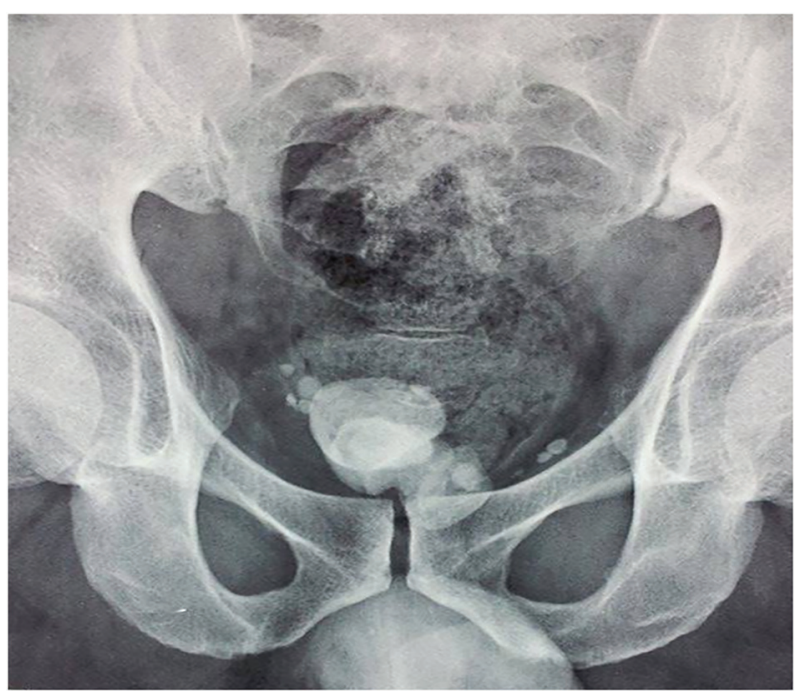

Figure 2. Retrograde urethrocystography (before contrast injection) showing recurrent prostatic urethral calculus.

evident urethral stenosis (Fig. 2). Complete blood count, renal function, serum parathyroid hormone, and serum calcium levels were within normal limits. An urethrocystoscopy showed normal urethra and a giant prostatic urethral calculus of uniform structure partially located in prostatic urethral diverticulum (Fig. 3). Because of the large stone burden, complete endoscopic removal was not feasible. Therefore, calculus was extracted entirely via cystotomy through bladder neck (Fig. 4). Postoperative plain radiography showed no residual calculus within the urinary tract. Infrared stone analysis revealed that it was composed of calcium phosphates.

\section{Discussion}

Prostatic parenchymal calculi also called "true prostatic calculi" are common, usually incidental, ?ndings on morphologic

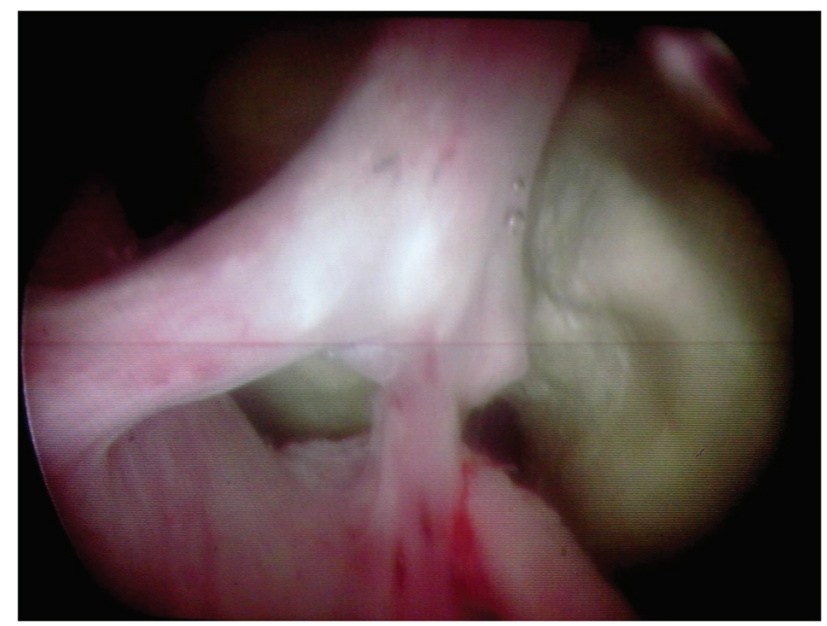

Figure 3. Urethroscopy: giant prostatic urethral calculus with prostate diverticulum.

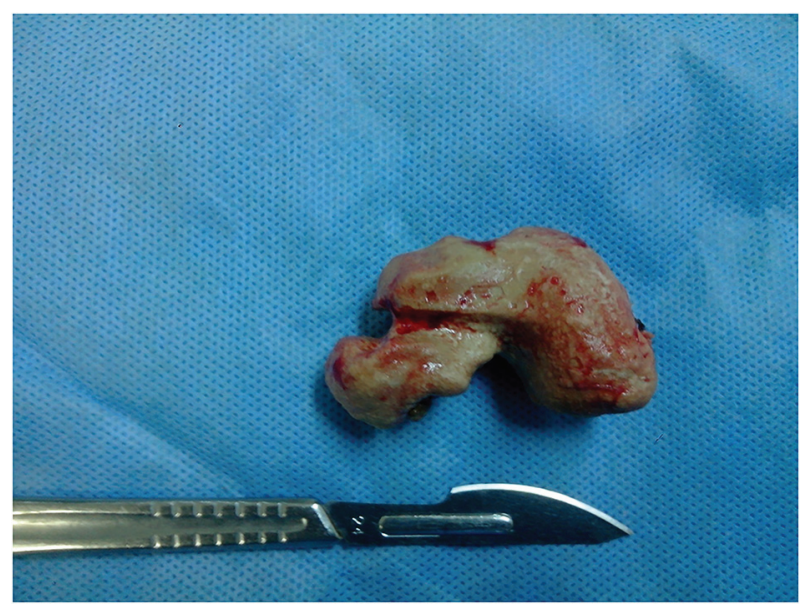

Figure 4. Recurrent uniform calculus extracted via cystotomy.

examinations in men older than 50 years. This entity should be not confused with primary calculi of the prostatic urethra which are exceedingly rare, especially giant ones. They occur more frequently in younger men [2].

The etiology of prostatic calculi remains unclear. True prostatic calculi are formed by the deposition of calcareous material on the corpora amylacea $[2,3]$. Prostatic urethral stones are classified as endogenous or primary (those formed de novo in the urethra) and exogenous or secondary (those formed in the upper urinary tract with secondary downward descent) [4, 5].

Primary native calculi are usually small and multiple, and secondary calculi are usually larger [4]. Primary urethral stones can be formed from direct precipitation of elements present in prostatic secretions stasis that results from obstruction, inflammation and chronic infection of the prostate ducts [6]. They are magnesium ammonium phosphate (struvite), calcium phosphate or calcium carbonate in composition, have no nucleus and are of uniform structure. They are formed in the urethra proximal to strictures, in congenital and acquired diverticula, with chronic infection with especially urea splitting organisms or with foreign bodies [5]. They generally do not cause acute symptoms because of their slow development.

Secondary or migrant stones are usually of calcium oxalate and phosphate [5]. They are much more common. They are most often encountered in association with urethral stricture disease or other forms of urethral obstruction. They often cause acute symptoms causing retention, frequency, dysuria, poor stream or dribbling [4].

We think that in the present case, the prostatic calculus is probably primary, due partially to the presence of prostatic diverticulum explaining recurrence.

Clinical examination is usually normal but sometimes these calculations are collected on digital rectal examination as a hard nodule that was suspected more of a prostate cancer. The diagnosis of prostatic calculi is radiological standard radiographs or ultrasound especially prostate transrectal which has better diagnostic sensitivity [6]. Retrograde cystography is indicated to detect associated urethral disease $[6,7]$. 
To date, there has been no consensus regarding the optimal treatment for giant prostatic urethral calculi. Management techniques have included radical prostatectomy, cystotomy with bladder neck incision, and endoscopic lithotripsy [8-10]. Vesical neck incision has been recommended as an adjunct to relieve obstruction and improve drainage of local infection [1]. Walker and Hamilton [10] reported the successful management of small urethral calculi by transurethral holmium laser ablation. This appears to be an attractive approach in the field of urology. Shah et al [1] were the first to report open prostatolithotomy as a novel method for the surgical extraction of giant prostatic calculi. The principles of this operation are based on simple retropubic prostatectomy, a well-known and effective way of enucleating the prostate for symptomatic gland hyperplasia. Most authors emphasize that giant urethral calculi are better treated with open surgery $[1,4]$.

Endoscopic screening of urethra should be performed before surgery to detect urethral obstruction or diverticula which may be misdiagnosed on morphologic examinations [9]. The diagnosis of prostatic urethral diverticulum was made during urethroscopy in our case.

Treatment and prevention of recurrent infections or prostatitis, treatment of concomitant abnormalities such as diverticula or urethral stenosis and metabolic evaluation with infrared stone analysis are recommended to prevent recurrence of this pathology.

\section{Conclusion}

Giant prostatic urethral calculus with prostate diverticulum is very rare. Endoscopic screening of urethra should be considered. The open surgery approaches are suggested for treatment management. However, transurethral holmium laser ablation is an attractive alternative that should be developed in the future. Metabolic evaluation of the patient and treatment of predisposing factors such as diverticula or urethral stenosis are recommended to prevent recurrence.

\section{Acknowledgments}

We thank Dr Mayssa Khribi for her English language assistance (Medical University of Sousse, Tunisia).

\section{Conflicts of Interest}

None.

\section{Funding Source}

None.

\section{References}

1. Shah SK, Chau MH, Schnepper GD, Lui PD. Open prostatolithotomy for the management of giant prostatic calculi. Urology. 2007;70(5):1008 e1009-1010.

2. Bedir S, Kilciler M, Akay O, Erdemir F, Avci A, Ozgok Y. Endoscopic treatment of multiple prostatic calculi causing urinary retention. Int J Urol. 2005;12(7):693-695.

3. Menon M, Resnick MI. Urinary lithiasis: etiology, diagnosis, and medical treatment. In: Walsh PC, Retik AB, Vaughan ED Jr, Wein AJ, Kavoussi LR, Novick AC, editors. Campbell's urology. 8th ed. Philadelphia: Saunders Elsevier; 2002; p. 3287-3289.

4. Prabhuswamy VK, Tiwari R, Krishnamoorthy R. A giant dumbbell shaped vesico-prostatic urethral calculus: a case report and review of literature. Case Rep Urol. 2013;2013:167635.

5. Kotkar K, Thakkar R, Songra M. Giant urethral calculus. J Surg Case Rep. 2011;2011(8):9.

6. Najoui M, Qarro A, Ammani A, Alami M. Giant prostatic calculi. Pan Afr Med J. 2013;14:69.

7. Mezzour MH, El Messaoudi YA, Balhouss H, Dakir M, Aboutaieb R, El Moussaoui A, Rabii R, Joual A, Meziane F. Grosse lithiase intra prostatique (A propos d'un cas). Afric J Urol. 2006;12:111-114.

8. Gawande AS, Kamat MH, Seebode JJ. Giant prostatic calculi. Urology. 1974;4(3):319-321.

9. Virgili G, Forte F, Sansalone S, Attisani F, De Carolis A, Di Stasi SM, Vespasiani G. Radical prostatectomy as unique chance for huge prostatic stones. Arch Ital Urol Androl. 2004;76(4):171-172.

10. Walker BR, Hamilton BD. Urethral calculi managed with transurethral Holmium laser ablation. J Pediatr Surg. 2001;36(9):E16. 